\title{
Nomogramas de ecuaciones de referencia espirométrica SER 2009
}

\author{
MÓNICA GUTIÉRREZ C.*, GONZALO VALDIVIA C.**, LUIS VILLARROEL DEL P.**, \\ GUSTAVO CONTRERAS T.***, CLAUDIA CARTAGENA S. $* * * *$ y CARMEN LISBOA B. $* * * * *$
}

\section{$\underline{\text { Spirometric nomograms for Chilean normal adults }}$}

As a result of the availability of programmes to detect and treat respiratory disease in Chile there has been a greater use of spirometry in diagnosis. To be able to correctly interpret spirometry results there must be reference patterns which, ideally come from the same population in which they will be used. In order to facilitate the use and application of new spirometric reference equations recently calculated for the adult population, there follows a series of nomograms. These nomograms have been designed for each of the following parameters: gender, age, and size of subjects between 19 and 50 years old and also for subjects over 51 years old. These equations are taken from 5 national studies. These studies included a population which complied with universal criteria to determine reference values ( 1.174 subjects, between 19 and 94 years old), spirometries were carried out on this population following international recommendations (ATS).

Key words: Spirometry, reference values, nomograms.

\section{Resumen}

La actual disponibilidad de programas de detección y tratamiento de enfermedades respiratorias en Chile ha determinado una mayor utilización de la espirometría en el diagnóstico de dichas enfermedades. La adecuada interpretación de los resultados de la espirometría requiere disponer de patrones de referencia idealmente originados en la misma población en la cual se utilizarán. Para facilitar el uso y aplicación de nuevas ecuaciones de referencia espirométricas recientemente modeladas en población adulta chilena, se presentan nomogramas diseñados para cada uno de los parámetros en base al género, edad y talla para sujetos entre 19 y 50 años y en mayores de 51 años. Estas ecuaciones derivan de 5 estudios nacionales que incluyeron población que cumplió criterios universales para determinar valores de referencia, (1.174 sujetos, 19 a 94 años), que efectuaron espirometrías siguiendo recomendaciones internacionales (ATS).

Palabras clave: Espirometría, valores de referencia, nomogramas.

\section{Introducción}

Durante los últimos años se ha asistido a una progresiva masificación en el uso clínico de la espirometría a nivel nacional, actualmente disponible en todos los establecimientos del sistema público de salud. Para su correcta utilización es indispensable disponer de adecuados valores de referencia, obtenidos de población chilena en base a procedimientos estandarizados ${ }^{1}$. Con esta finalidad, la Comisión de Función Pulmonar de la Sociedad Chilena de Enfermedades Respiratorias propuso a su Directorio obtener ecuaciones de referencia espirométricas representativas de la población general sana chilena, propósito recientemente logrado. Con el objetivo de favorecer la aplicación expedita de estas ecuaciones, al igual que en el estudio platino ${ }^{2}$, se decidió diseñar no-

\footnotetext{
* Hospital Naval Viña del Mar.

** Departamento de Salud Pública. Pontificia Universidad Católica de Chile.

*** Asociación Chilena de Seguridad.

**** Hospital Van Buren, Valparaíso.

***** Departamento de Enfermedades Respiratorias. Pontificia Universidad Católica de Chile.
} 
mogramas que faciliten su uso en la práctica clínica, los que se presentan en esta comunicación.

\section{Material y Método}

En base a las ecuaciones obtenidas recientemente en población adulta chilena entre 19 y 94 años, cuyas características se describen en la Tabla 1, se diseñaron nomogramas separados según género y grupos de edad.

\section{Diseño del Nomograma}

El valor teórico de cada variable de función pulmonar fue obtenido a partir del diseño de diversas ecuaciones lineales (Tabla 2), en las cuales dicha variable corresponde a:

Variable $=$ Intercepto $+\left(\mathrm{B}^{*}\right.$ edad $)+\left(\mathrm{C}^{*}\right.$ talla $)$

Utilizando el sistema de resolución de ecuaciones lineales se puede graficar un modelo con la forma de un cuadrado, donde cada lado vertical

Tabla 1. Características de las poblaciones de los estudios de base para la elaboración de ecuaciones de referencia espirométricas

\begin{tabular}{|llccl|}
\hline Autores & Población & $\mathbf{1 9}$ a 50 años & $\mathbf{5 0}$ años & Espirómetros \\
\hline Gutiérrez y cols $1996^{5}$ & $\begin{array}{l}\text { Viña del Mar } \\
\text { Valparaíso }\end{array}$ & 326 & 73 & Jaeger PFT System \\
\hline Lisboa* & Santiago & 3 & 95 & Fukuda® 250 \\
\hline Cartagena* & Valparaíso & 0 & 45 & Sibel \\
\hline PLATINO & Santiago & 41 & 91 & Easy one de NDD \\
\hline Contreras* & Santiago & 436 & 64 & Medgraphics \\
\hline
\end{tabular}

*Bases de datos no publicadas aportadas por los autores.

Tabla 2. Parámetros estadísticos derivados de ecuaciones lineales para hombres y mujeres a partir de medidas espirométricas seleccionadas según género y edad

\begin{tabular}{|c|c|c|c|c|}
\hline & Intercepto & $\begin{array}{l}\text { Constante } \\
\text { Talla }(\mathrm{cm})\end{array}$ & $\begin{array}{c}\text { Constante } \\
\text { Edad (años) }\end{array}$ & Percentil 5 (\%) \\
\hline \multicolumn{5}{|c|}{ Mujeres 19 a 50 años } \\
\hline CVF (L) & $-2,418$ & 0,04089 & $-0,01$ & 81 \\
\hline $\mathrm{VEF}_{1}(\mathrm{~L})$ & $-1,328$ & 0,03159 & $-0,018$ & 81 \\
\hline $\mathrm{VEF}_{1} / \mathrm{CVF}(\%)$ & 102,411 & $-0,06913$ & $-0,251$ & 89 \\
\hline $\mathrm{FEF}_{25-75}(\mathrm{~L} / \mathrm{s})$ & 1,887 & 0,01662 & $-0,035$ & 64 \\
\hline \multicolumn{5}{|c|}{ Mujeres mayores de 50 años } \\
\hline CVF (L) & $-1,301$ & 0,03597 & $-0,021$ & 76 \\
\hline $\mathrm{VEF}_{1}(\mathrm{~L})$ & $-0,32$ & 0,026 & $-0,023$ & 74 \\
\hline $\mathrm{VEF}_{1} / \mathrm{CVF}(\%)$ & 100,661 & $-0,04895$ & $-0,275$ & 88 \\
\hline $\mathrm{FEF}_{25-75}(\mathrm{~L} / \mathrm{s})$ & 2,181 & 0,01533 & $-0,041$ & 36 \\
\hline \multicolumn{5}{|c|}{ Hombres 19 a 50 años } \\
\hline CVF (L) & $-6,525$ & 0,07084 & $-0,012$ & 81 \\
\hline $\mathrm{VEF}_{1}(\mathrm{~L})$ & $-3,499$ & 0,04957 & $-0,024$ & 81 \\
\hline $\mathrm{VEF}_{1} / \mathrm{CVF}(\%)$ & 119,282 & $-0,16819$ & $-0,274$ & 88 \\
\hline $\mathrm{FEF}_{25-75}(\mathrm{~L} / \mathrm{s})$ & 1,892 & 0,0231 & $-0,047$ & 60 \\
\hline \multicolumn{5}{|c|}{ Hombres mayores de 50 años } \\
\hline CVF (L) & $-3,072$ & 0,05555 & $-0,033$ & 77 \\
\hline $\mathrm{VEF}_{1}(\mathrm{~L})$ & $-1,676$ & 0,04002 & $-0,031$ & 75 \\
\hline $\mathrm{VEF}_{1} / \mathrm{CVF}(\%)$ & 87,278 & $-0,00672$ & $-0,181$ & 86 \\
\hline $\mathrm{FEF}_{25-75}(\mathrm{~L} / \mathrm{s})$ & $-1,07$ & 0,0351 & $-0,035$ & 33 \\
\hline
\end{tabular}


corresponde a un eje, indicando el eje izquierdo a la variable edad expresada en años $\left(\mathrm{W}_{1}\right.$ a $\left.\mathrm{W}_{2}\right)$ y el del costado derecho, a la talla expresada en $\mathrm{cm}\left(Z_{1}\right.$ a $\left.Z_{2}\right)$.

La altura de cada eje es igual a la distancia que los separa y corresponde a "Y".

Las variables de función pulmonar incluidas Capacidad vital forzada (CVF), Volumen espiratorio forzado en el primer segundo $\left(\mathrm{VEF}_{1}\right)$, relación $\mathrm{VEF}_{1} / \mathrm{CVF}$ y Flujo espiratorio forzado entre el 25 y $75 \%$ de la CVF $\left(\mathrm{FEF}_{25-75}\right)$ corresponden a ejes verticales y paralelos a este cuadrado.

Utilizando la siguiente ecuación ${ }^{3}$ para cada variable en cada grupo:

$$
X=\frac{Y\left(Z_{1}-Z_{2}\right)}{B / C\left(W_{1}-W_{2}\right)+\left(Z_{1}-Z_{2}\right)}
$$

Donde:

$\mathrm{Z}_{1}$ : límite superior del eje de la talla (media + 2DS)

$\mathrm{Z}_{2}$ : límite inferior del eje de la talla (media - 2DS)

B: constante de edad
C: constante de talla

$\mathrm{W}_{1}$ : límite superior del eje de la edad

$\mathrm{W}_{2}$ : límite inferior del eje de la edad

Se obtiene la incógnita $\mathbf{X}$ que corresponde a la distancia del 0 en la línea horizontal en que se ubica el eje de esa variable. El 0 esta determinado por el punto en que el eje de la edad corta la línea horizontal. Para obtener el valor del límite superior e inferior de cada variable, se aplica la ecuación correspondiente para esa edad y estatura.

Se usó test t de Student para muestras pareadas y gráficos de Bland-Altman ${ }^{3}$ para comparar los valores arrojados por los nomogramas y los obtenidos aplicando las ecuaciones lineales.

\section{Resultados}

Se diseñaron nomogramas para el cálculo de Capacidad vital forzada (CVF), Volumen espiratorio forzado en el primer segundo $\left(\mathrm{VEF}_{1}\right)$, relación $\mathrm{VEF}_{1} / \mathrm{CVF}$ y Flujo espiratorio forzado entre el 25 y $75 \%$ de la CVF $\left(\mathrm{FEF}_{25-75}\right)$ para:

Mujeres entre 19 y 50 años (Figura 1) y entre 51 y 94 años (Figura 2).

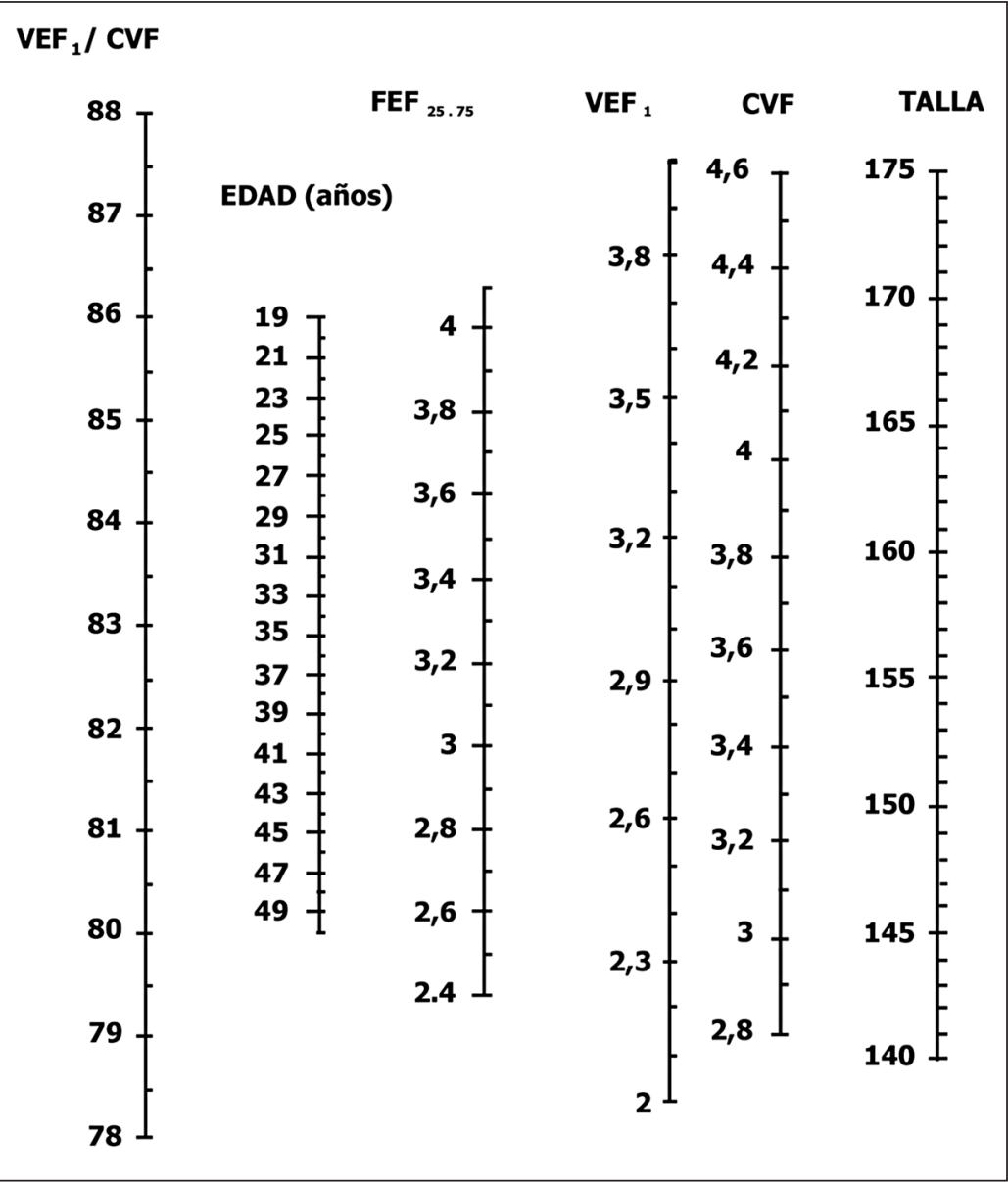

Figura 1. Nomograma donde se relaciona cada variable espirométrica con la edad y talla en $\mathrm{cm}$ en mujeres de 19 a 50 años. Las unidades para las variables espirométricas son las señaladas en la Tabla 2. 


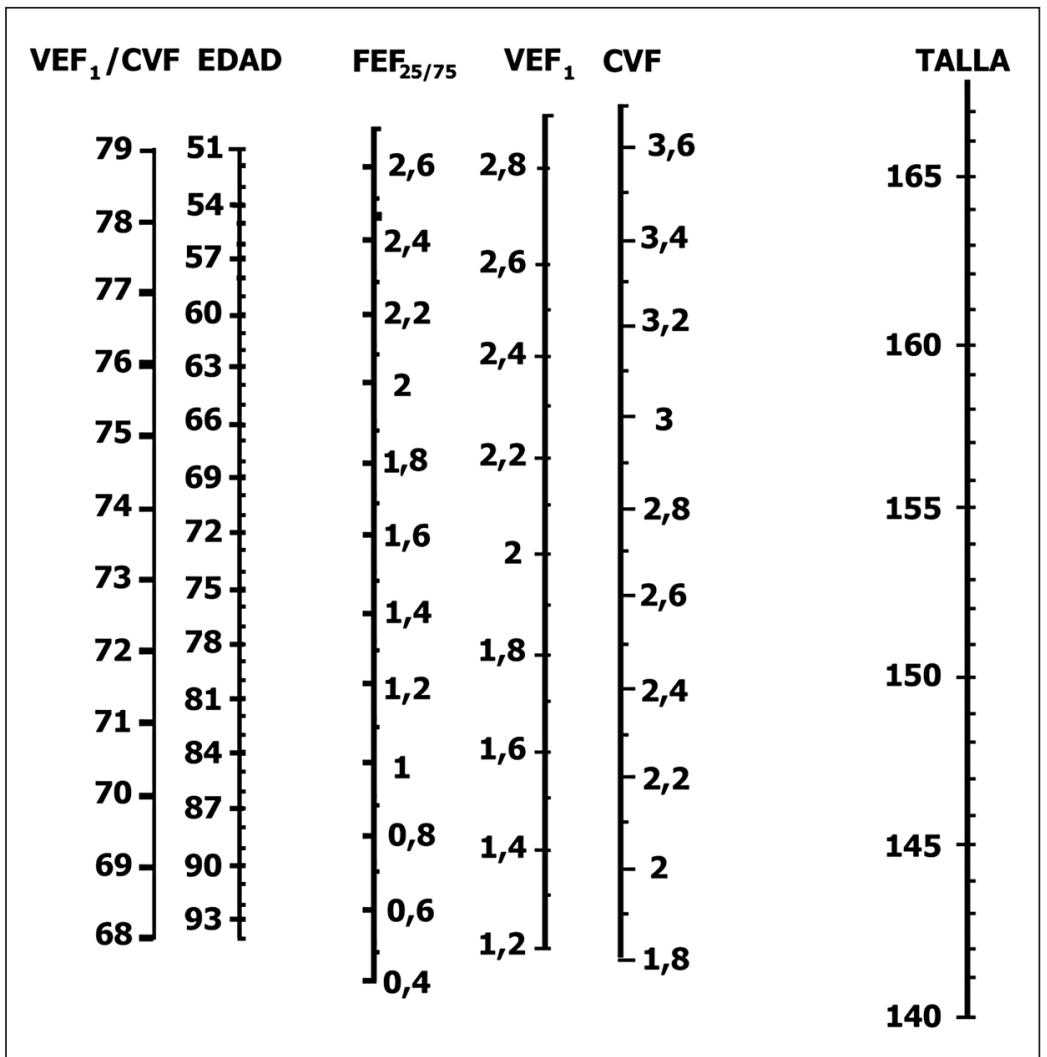

Figura 2. Nomograma donde se relaciona cada variable espirométrica con la edad en años y talla en $\mathrm{cm}$ en mujeres de 51 a 94 años. Las unidades para las variables espirométricas son las señaladas en la Tabla 2.

Tabla 3. Comparación lectura de nomograma versus resolución ecuación

\begin{tabular}{|c|c|c|c|c|c|c|c|c|}
\hline \multirow{3}{*}{ Ejemplos } & \multicolumn{2}{|c|}{ CVF (L) } & \multicolumn{2}{|c|}{$\mathrm{VEF}_{1}(\mathrm{~L})$} & \multicolumn{2}{|c|}{$\mathrm{VEF}_{1} / \mathrm{CVF}(\%)$} & \multicolumn{2}{|c|}{$\mathrm{FEF}_{25-75}(\mathrm{~L} / \mathbf{s})$} \\
\hline & $\mathbf{N}$ & $\mathbf{E}$ & $\mathbf{N}$ & $\mathbf{E}$ & $\mathbf{N}$ & $\mathbf{E}$ & $\mathbf{N}$ & $\mathbf{E}$ \\
\hline & & & & & & & & \\
\hline \multicolumn{9}{|c|}{ Mujeres $<51$ años } \\
\hline 43 años $162 \mathrm{~cm}$ & 3,77 & 3,78 & 3,01 & 3,02 & 80,60 & 81,00 & 3,09 & 3,07 \\
\hline 28 años $143 \mathrm{~cm}$ & 3,16 & 3,15 & 2,70 & 2,69 & 85,60 & 85,00 & 3,29 & 3,28 \\
\hline 29 años $153 \mathrm{~cm}$ & 3,55 & 3,55 & 2,98 & 2,98 & 84,60 & 85,00 & 3,41 & 3,41 \\
\hline \multicolumn{9}{|c|}{ Mujeres $>\mathbf{5 0}$ años } \\
\hline 58 años $149 \mathrm{~cm}$ & 2,80 & 2,84 & 2,20 & 2,22 & 78,00 & 77,00 & 2,08 & 2,09 \\
\hline 60 años $167 \mathrm{~cm}$ & 3,44 & 3,43 & 2,64 & 2,63 & 76,00 & 76,00 & 2,25 & 2,27 \\
\hline 78 años $155 \mathrm{~cm}$ & 2,64 & 2,64 & 1,92 & 1,92 & 72,00 & 72,00 & 1,35 & 1,36 \\
\hline \multicolumn{9}{|c|}{ Hombres $<51$ años } \\
\hline 33 años $178 \mathrm{~cm}$ & 5,60 & 5,65 & 4,52 & 4,53 & 80,00 & 80,00 & 4,50 & 4,45 \\
\hline 45 años $165 \mathrm{~cm}$ & 4,60 & 4,59 & 3,60 & 3,60 & 79,00 & 79,00 & 3,60 & 3,59 \\
\hline 37 años $152 \mathrm{~cm}$ & 3,80 & 3,75 & 3,15 & 3,14 & 83,30 & 84,00 & 3,70 & 3,66 \\
\hline \multicolumn{9}{|c|}{ Hombres $>50$ años } \\
\hline 57 años $152 \mathrm{~cm}$ & 3,45 & 3,49 & 2,66 & 2,64 & 76,00 & 76,00 & 2,30 & 2,27 \\
\hline 78 años $164 \mathrm{~cm}$ & 3,44 & 3,46 & 2,48 & 2,47 & 72,00 & 72,00 & 1,95 & 1,96 \\
\hline 66 años $175 \mathrm{~cm}$ & 4,45 & 4,47 & 3,29 & 3,28 & 74,40 & 74,00 & 2,78 & 2,76 \\
\hline $\mathrm{p}$ & \multicolumn{2}{|c|}{0,323} & \multicolumn{2}{|c|}{0,463} & \multicolumn{2}{|c|}{0,759} & \multicolumn{2}{|c|}{0,115} \\
\hline
\end{tabular}

$\mathrm{N}=$ Valor obtenido de la lectura del nomograma. $\mathrm{E}=$ Valor obtenido por la resolución de la ecuación. 


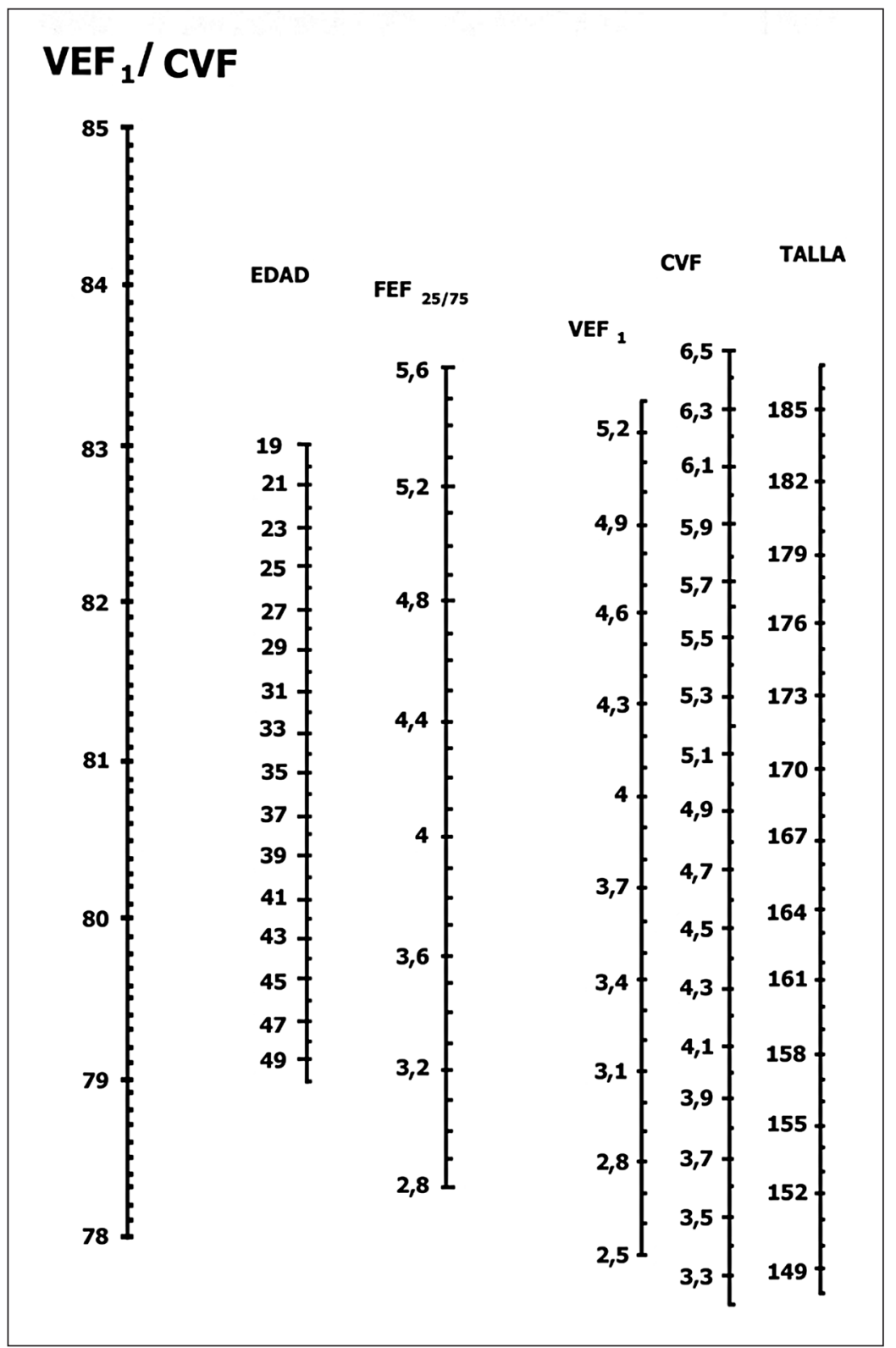

Figura 3. Nomograma donde se relaciona cada variable espirométrica con la edad en años y talla en cm en hombres de 19 a 50 años. Las unidades para las variables espirométricas son las señaladas en la Tabla 2.

Hombres entre 19 y 50 años (Figura 3) y entre 51 y 84 años (Figura 4).

Se comparó, mediante test $\mathrm{t}$ de Student para muestras pareadas, la lectura del nomograma para cada variable versus el valor entregado al resolver la ecuación, no encontrando diferencias clínicas ni estadísticamente significativas $(\mathrm{p}>0,05)$ en los 4 nomogramas diseñados (Tabla 3). También se realizaron gráficos de Bland-Altman para detectar la existencia de algún patrón en las diferencias observadas en los valores obtenidos al aplicar el nomograma y la correspondiente ecuación (Figura 5). Con la excepción del gráfico de $\mathrm{FEF}_{25-75}$, en que se observa valores algo mayores en el nomograma (aunque sin diferencias estadísticamente significativas), el resto de los parámetros de función pulmonar se comportan de forma similar tanto para el nomograma como en los valores obtenidos directamente a partir de las ecuaciones. 


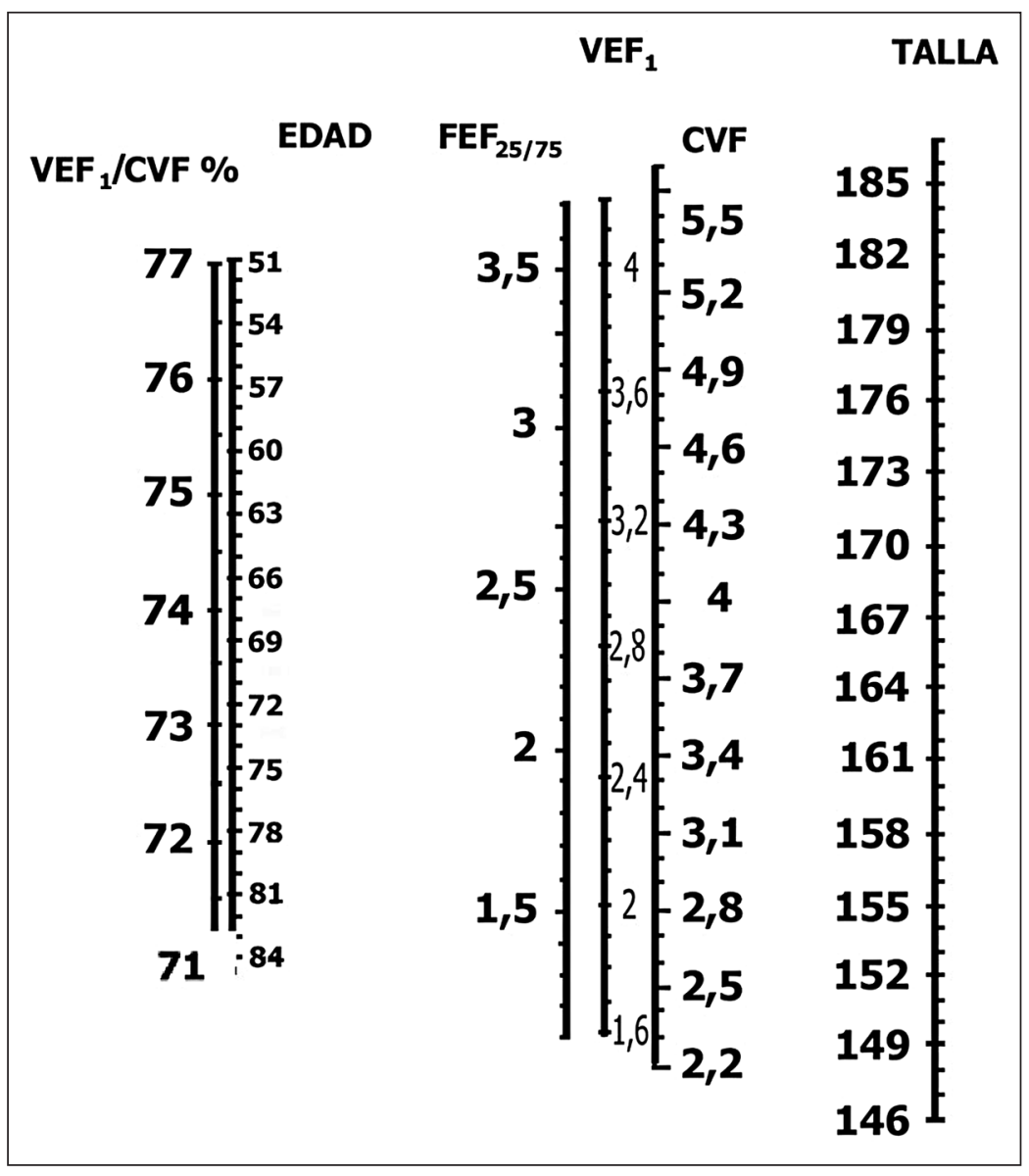

Figura 4. Nomograma donde se relaciona cada variable espirométrica con la edad en años y talla en cm en hombres de 51 a 84 años. Las unidades para las variables espirométricas son las señaladas en la Tabla 2.
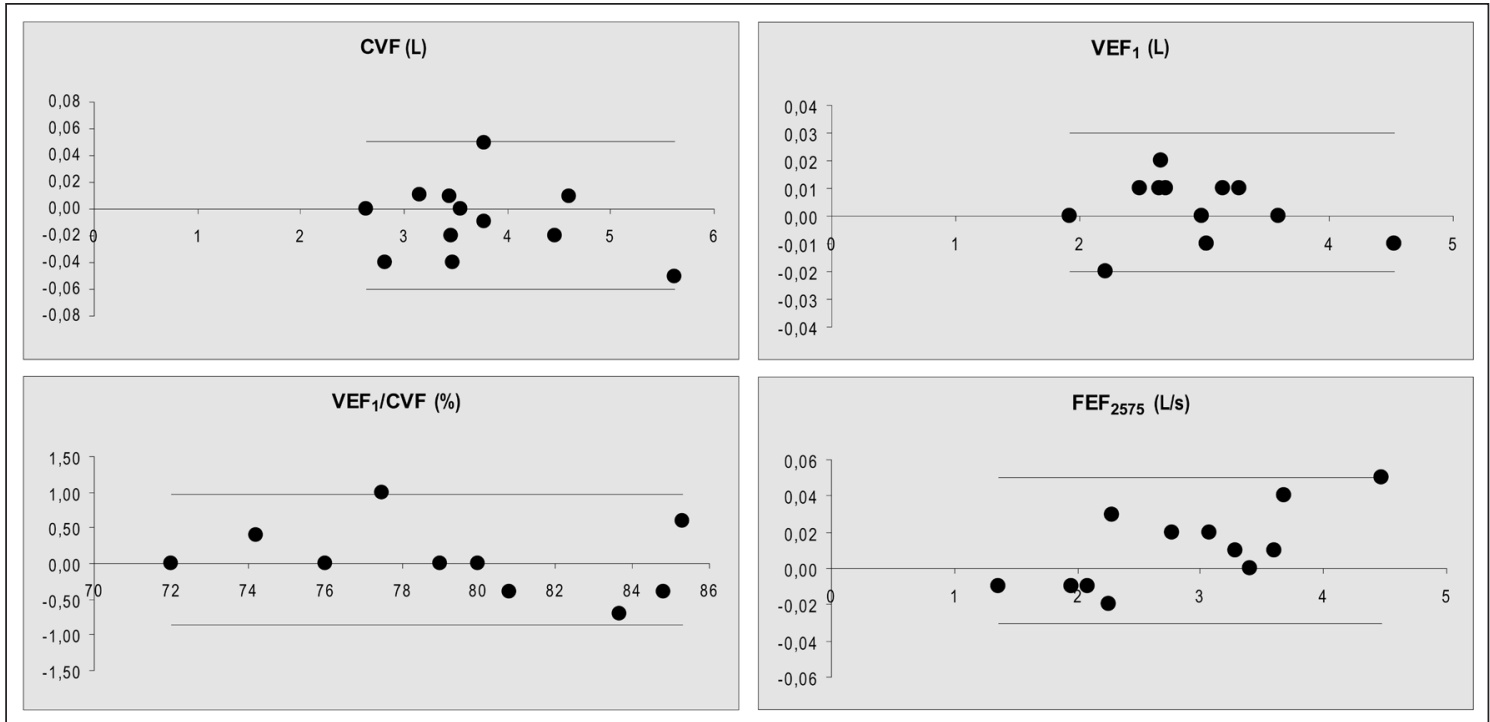

Figura 5. Gráficos de Bland y Altman para $\mathrm{VEF}_{1}, \mathrm{CVF}, \mathrm{FEF}_{25-75}$ y CVF para valores teóricos obtenidos a partir de Nomogramas y ecuaciones. El eje "X" representa el valor promedio de cada par de valores obtenidos del nomograma y de la ecuación, y el eje "Y", la diferencia observada entre el valor del nomograma y la ecuación. Las líneas horizontales corresponden a un intervalo de $95 \%$ de confianza para la diferencia. 


\section{Conclusiones}

Se logró obtener nomogramas confiables para el cálculo del valor teórico esperado para cada variable espirométrica, los que no difieren del valor entregado por la ecuación; esto es de particular importancia considerando que aún las ecuaciones calculadas no están incorporadas en el software correspondiente de los espirómetros.

Para su utilización simplemente se necesita un lápiz y una regla. Se debe trazar una recta entre la edad y la talla, obteniendo el valor de cada parámetro en el punto en que se cruza esta recta.

\section{Agradecimientos}

Los autores agradecen al Sr. Jorge Gutiérrez por el diseño de la fórmula matemática para graficar los nomogramas y su valiosa ayuda en aplicarla.

\section{Bibliografía}

1.- PELlEGRINO R, VIEGI G, BRUSASCO V, CRAPO R O, BURGOS F, CASABURI R, et al. Interpretative strategies for lung function test. Eur Respir J 2005; 26: 948-68.

2.- PÉREZ-PADILLA R, VALDIVIA G, MUIÑO A, LÓPEZ M, MÁRQUEZ M, MONTES DE OCA M, et al. Valores de referencia espirométrica en cinco grandes ciudades de Latinoamérica para sujetos de 40 o más años de edad. Arch Bronconeumol 2006; 42: 317-25.

3.- GUTIÉRREZ M, RIOSECO F, ROJAS A, CASANOVA D. Ecuaciones de referencia espirométrica en población chilena. Rev Chil Enf Respir 1997; 13: 165-77.

4.- BLAND J M, ALTMAN D G. Statistical methods for assessing agreement between two methods of clinical measurement. Lancet 1986; 327 (8476): 307-10.

5.- GUTIÉRREZ M, RIOSECO F, ROJAS A, CASANOVA D. Determinación de valores espirométricos en una población chilena normal mayor de 5 años, a nivel del mar. Rev Med Chile 1996; 124: 1295-306.
Correspondencia a:

Dra. Mónica Gutiérrez Clavería

Hospital Naval Viña del Mar

Jefatura del Laboratorio de Función Pulmonar

Docente ad honorem Universidad de Valparaíso

5 norte 1011 dpto 71 Viña del Mar

Teléfono: 322471603

E-mail: mgancaroe@gmail.com 